\title{
Smoothing Filters for Waveform Image Segmentation
}

\author{
Kedir Kamu Sirur, Ye Peng, and Zhang Qinchuan
}

\begin{abstract}
In this paper, we studied the impact of smoothing filters when applied to waveform image preprocessing for segmentation. To find out the characteristics of the output, we made an experiment on both real time and synthetic images. During our experiment, we have considered frequency-domain waveform images, time-domain waveform images, and ordinary test images. All the sample images are converted to gray level image and then smoothed with smoothing spline functions. We applied both global filter and localized filters. Finally, all the images are segmented using Intersecting Cortical Model (ICM) algorithm. The result of the experiment is evaluated using Peak Signal to Noise Ratio (PSNR), Mean Squared Error (MSE) and Visual Comparison methods. This research found out that we should be selective when we use smoothing filters on waveform images because of the unique structural behavior and high sensitivity of waveform images. The results proved that using global smoothing filters for waveform image preprocessing has a drastic negative effect and can change the pertinent signals. On the other hand, the use of localized smoothing filters resulted in sounding segmentation result from image processing point of view. This study also proved the increased performance of localized smoothing filters when applied to frequency-domain waveform images than time-domain waveform images. Thus we propose to avoid using global smoothing parameters for preprocessing of waveform images for segmentation and strongly recommend using localized smoothing filters.
\end{abstract}

Index Terms-Global parameter, local parameter, segmentation, smoothing, waveform image.

\section{INTRODUCTION}

A signal is a physical phenomenon that is a function of time, this describes what is being measured and as will be seen, is distinguished from a waveform. And the Waveforms are the things that are actually observed, acquired and analyzed [1]. During analysis of the signals to extract the pertinent information encoded on the waveform images we may need preprocessing to clean the noises. The problem of image recovering is to reduce undesirable distortions and noise while preserving important features such as homogeneous regions, discontinuities, edges and textures [2].

There are various noise removal methods which can satisfy the specific needs of different applications or perceptions with defined constraints. Since images are easily prone to various types of distortions, enhancing the images before analysis is very important. Image enhancement techniques improve the quality of the image as perceived by human and

Manuscript received July 24, 2017; revised October 13, 2017. This work was supported by the National Natural Science Foundation of China (Grant No. 61301263 and 61701077), and the Science and Technology Project of Sichuan Province (Grant No. 2016JY0103).

Kedir Kamu Sirur, Ye Peng, and Zhang Qinchuan are with the University of Electronic Science and Technology of China, Chengdu, Sichuan, P. R. China (e-mail: kedirkan@yahoo.com, \{yepeng,zhangqc\}@uestc.edu.cn). used to get details that are obscured or to highlight certain features of interest in the image for further processing. Image smoothing algorithms offer a wide variety of approaches to modify images to achieve visually acceptable ones.

The image enhancement can be done either through spatial domain or frequency domain. The choice of such techniques is a function of the specific task, image content, observer characteristics, and viewing conditions [3]. In the spatial domain, we have two important methods, point processing, and neighborhood processing, which are considered to be suitable for real time image manipulation [3], [4]. In neighborhood processing method, an image is enhanced by applying a mathematical function to each pixel in an image along with its neighboring pixels.

The two main aspects that are considered to be essential in image enhancement are image sharpening and image smoothing. In the image sharpening, image information is detailed in such a way that it contains high spatial components of the image. In image smoothing, image information is detailed in such a way that it contains low spatial components of the image. Smoothing filters are capable of blurring and reduce noises. Blurring is at preprocessing stage to remove fine details which are not important for object extraction and to the bridging of small gaps in lines and curves [5], [6]. Smoothing some pixel intensities might suppress, average out the intensity by the values of the neighborhood and shifted to the other intensity level, which could lead to erroneous segmentation result on very sensitive images.

So the unique structure of waveform images and their high sensitivity to changes in the pixel values of the image during processing inspired us to research the ordinary recommendations to use smoothing filter before segmentation of Waveform images.

The rest of the paper is organized as follows; Section II presents a review of smoothing filters, Section III elaborates the experimental setup and Section IV discusses the findings of the experiment. Finally, Section V presents the conclusions of the research.

\section{SMOOTHING FILTERS}

Gaussian filters are the only ones which are separable and at least to a lattice approximation, circularly symmetric. They also overcome the drawbacks of moving average filters because of weights decay to zero [3], [5]. A pixel the farther away from the neighbors, the smaller the weight will be. As with all smoothing operations, there is the fundamental trade-off between variance and bias: a filter which operates in a large neighborhood will be more effective at reducing noise but will also blur edges [3]. Edge-preserving smoothing filters are much more suitable for feature extraction. The nonlinear algorithms calculate the filtered gray value in dependence of 
the content of a defined neighborhood. From the list of the neighborhood pixels, only these are taken for the averaging, which have similar gray values compared to the pixel in consideration. Each edge-preserving filter has its own specific algorithm, but they all have in common, that the effect of this smoothing strategy is to preserve edges. Unfortunately, these smoothing filters have the characters not to smooth satisfyingly, because small gray value fluctuations existing in the really homogeneous areas are emphasized and not reduced [7].

Smoothing splines, like kernel regression and k-nearest-neighbors regression, provide a flexible way of estimating the underlying regression function. Also, smoothing splines are generally much more computationally efficient, simple and numerically accurate [8]. In words a $\mathrm{k}^{\text {th }}$ order spline is a piecewise polynomial function of degree $\mathrm{k}$, that is continuous and has continuous derivatives of orders 1 to $\mathrm{k}-1$, at its knot points. A cubic spline is piecewise cubic functions that are continuous and have continuous first, and second derivatives and their continuity in all of their lower order derivatives make splines very smooth and ideal [8], [9]. The above mentioned unique characteristic of smoothing splines makes them much more convenient to be augmented with cortical models to segment the natural signal representation image [10].

Natural image signals are highly structured: their pixels exhibit strong dependencies, especially when they are spatially proximate. These dependencies carry important information about the structure of the objects in the visual scene. The luminance of the surface of an object being observed is the product of the illumination and the reflectance, but the structures of the objects in the scene, are independent of the illumination [11], [12].

Prabha et al. [3] recommended conservative smoothing and adaptive median filters respectively among the neighborhood processing algorithms for blur effect removal at a low level because of their capability to preserve detailed information as a result of their ability to identify noisy pixels from noise-free pixels. Cheng et al. [2] proposed a method to remove the effect of smoothing filters on color images segmentation using a single colored mask. Chen et al. proposal is on the top of Yang et al. [13] method, in which to adopt a single colored mask for color adjustment. Since Yang et al. method have removed the effect of central pixels in a mask on a mask calculations the augmentation of Chen et al. proposal can clear the general effect of smoothing filters on color image segmentation. A number of methods have been developed that achieve adaptive smoothing by appropriately weighting the intensities within the window. Goshtasby et al. [6] introduced an adaptive image smoothing algorithm in which the windows shape, size, and orientation vary with the image structure and most smoothing is done in the direction of least gradient. Energy minimizing and optimization approaches to adaptive smoothing have been proposed. In an Energy minimizing model, Kervrann [14] keeps the window shape square but allows the window size to vary. In this model at each pixel, a regression function is estimated by iteratively growing the window size and adaptively weighting the image intensities until a minimum is reached in an energy function.

\section{EXPERIMENT}

To analyze the impact of using smoothing filters on segmentation of waveform images we conducted the experiments on real time images and synthetic images. The images are of two categories. The first type of images used is natural images, which can be an example of ordinary images. These images are differing both in structure and texture from waveform images. The second type of images is waveform images, these images are also from two categories real-time images captured from the screen of the oscilloscope and synthetic images. The images were also subjected to various levels of natural and synthetic noises.

During the experiments, all images are converted to gray level first. Then the images are subjected to smoothing with both localized adaptive operators and global smoothing operators. Finally, all images are segmented using both Pulse PCNN and ICM. To smooth the images we used B-Spline smoothing function, which is much more computationally efficient, numerically accurate and simple [8], [9].

Smoothing splines are robust in the presence of noise and real-time segmentation is achievable. Also, the smoothing spline function used is able to remove single pixel noises which guarantee better segmentation for such very sensitive images [8]. During the segmentation process, we have used both PCNN and ICM. But since Intersecting Cortical Model has performed better on the segmentation, we opted to analyze only the result of ICM segmentation algorithm. Finally to compare the result we have calculated the PSNR and MSE for each segmented image [12], [15]-[17]. Visual comparison, PSNR, MSE are used to evaluate the result of segmentation and draw our conclusion from image processing point of view. MSE objectively quantifies the strength of the error signal which is based on the assumption that the loss of perceptual quality is directly related to the visibility of the error signal. An image signal whose quality is being evaluated can be thought of as a sum of an undistorted signal and an error signal [12].

\section{RESULT AND DISCUSSION}

The use of smoothing B-Spline operators on natural test images has shown the positive response for both local smoothing spline parameters and global smoothing parameters see Fig. 1, see Fig. 2 and see Table I. Outperforming result is achieved with global smoothing filters at all levels of noise for ordinary test images. As we can see from the segmentation results for ordinary images, the images smoothed before segmentation using global smoothing parameters has resulted in an appealing segmented region from image processing point of view. It's also apparent when we compare visually the segmentation result using global parameters has resulted in clearly separated regions which are an appealing result from image processing point of view than the other. Images smoothed with local Spline parameters also generated visually appealing results, but the unnecessary details such as cap details and hair details are included in the segment that can hinder the feature extraction process. The tabulated quality measures also demonstrate the better performance of global smoothing filters for ordinary 
images.

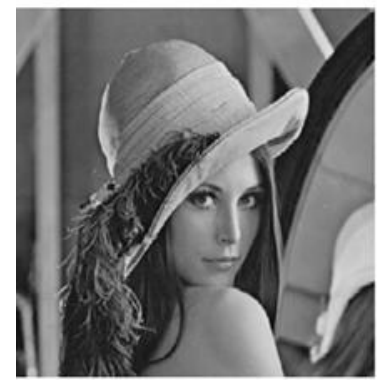

a

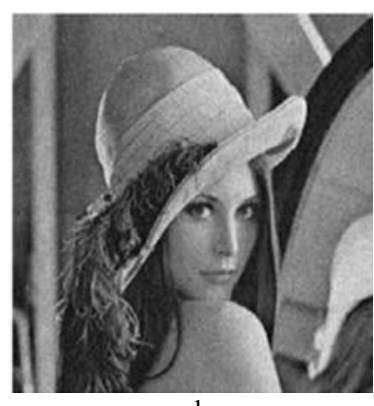

b
Fig. 1. (a) Lena test image. (b) Lena Test Image corrupted with Gaussian noise of Zero mean with standard deviation of 50 .

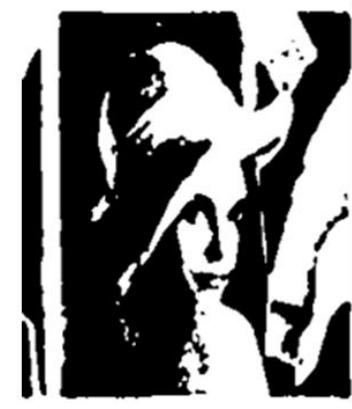

a

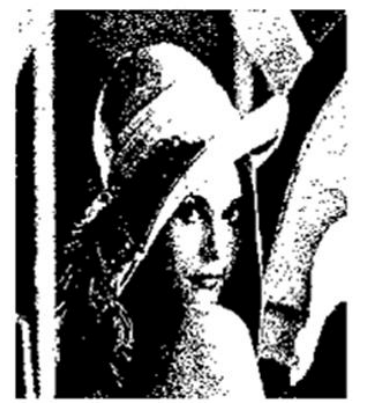

Fig. 2. (a) (b) Corrupted Lena test image in Fig. (1b) segmented after smoothed globally and locally, respectively.

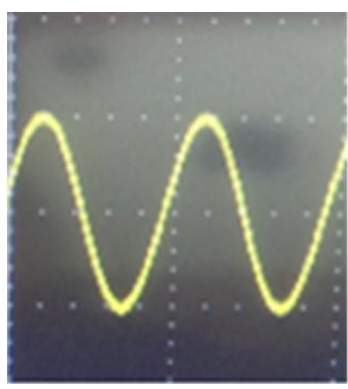

a

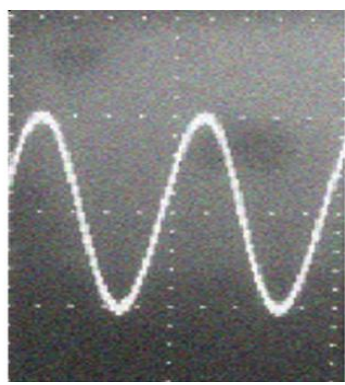

b
Fig. 3. (a) Time domain waveform image corrupted with non-uniform illumination. (b) Image (a) converted to gray scale, corrupted with non-uniform illumination and Gaussian noise of zero-mean and variance of 0.0025 .

The response of waveform images for smoothing filters is different than ordinary images. See Fig. 3b, see Fig. 4a, see Fig. 5, see Fig. 6a, and see Fig. 7a show the input and the response to global smoothing filter which resulted in irrelevant shades as the border of the image and replicated waveforms as well. We can see that waveforms are also merged to one, forming a shaded area which is significant to change the information content and lead to erroneous interpretation. The quality measures tabulated clearly demonstrate the negative impact of the global operation on the segmentation process. As we can observe in Table VI the PSNR is reduced by 15.77 and the MSE increased by 0.96 when we smooth it by the global operator instead of using a localized operator, which is a drastic effect on the quality of the segmented image. See Table II and see Table III also presents clearly how the quality metrics are negatively affected. See Fig. 8 when the image complexity of waveform increases and smoothed with global parameters, the PSNR is increased by 6.0005 when smoothed by local parameter than when smoothed with global and MSE is reduced by 0.1337 , which is very significant, see Table IV, see Table $\mathrm{V}$, and see Table VI. The experimental results clearly demonstrate using localized smoothing filter outperforms using a global operator, also proves the serious impact of using global operators. See Fig. 4b, see Fig. 6b, see Fig. 7b, see Fig. 8b, see Fig. 9, see Fig. 10a, and see Fig. 11a all presents the response to localized filters which demonstrates neat, complete and visually appealing segmentation results, which sounds great from image processing point of view.

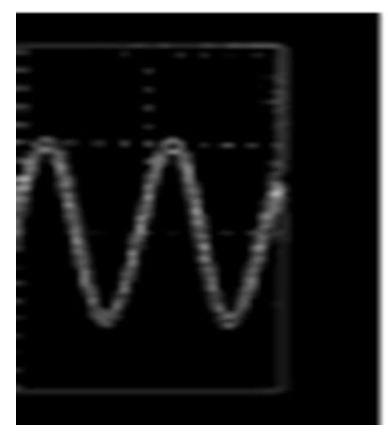

a

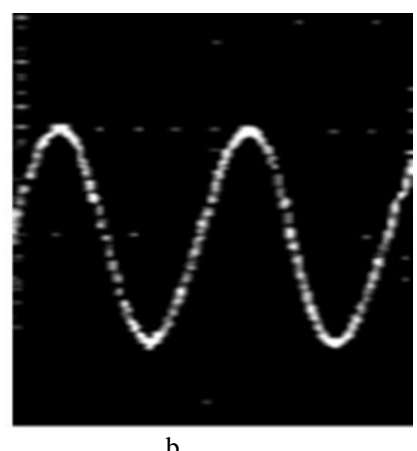

Fig. 4. (a) (b) Segmented Time domain waveform image in fig. (3b) after smoothed with global and local parameters respectively.

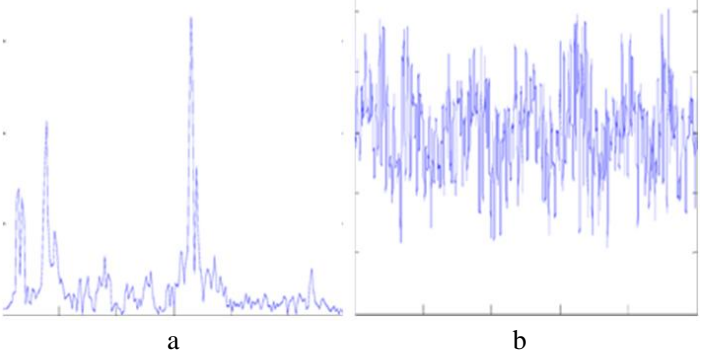

Fig. 5. (a) Frequency-domain waveform image. (b) Time-domain waveform image.

TABLE I: PSNR AND MSE VALUES OF SEGMENTED LENA TEST IMAGE AFTER SMOOTHED WITH GLOBAL AND LOCAL PARAMETERS

\begin{tabular}{|c|c|c|c|c|}
\hline \multirow{2}{*}{$\begin{array}{c}\text { Noise } \\
\text { level }\end{array}$} & \multicolumn{2}{|c|}{$\begin{array}{c}\text { Smoothed using global } \\
\text { parameter }\end{array}$} & \multicolumn{2}{|c|}{$\begin{array}{c}\text { Smoothed using local } \\
\text { parameter }\end{array}$} \\
\cline { 2 - 5 } & PSNR & MSE & PSNR & MSE \\
\hline $\begin{array}{c}\text { Noise } \\
\text { free }\end{array}$ & 51.7053 & 0.4391 & 51.7496 & 0.4346 \\
\hline 50 & 50.5357 & 0.5748 & 50.5674 & 0.5706 \\
\hline
\end{tabular}

TABLE II: PSNR AND MSE VALUES OF SEGMENTED TIME-DOMAIN WAVEFORM IMAGE CORRUPTED WITH NON-UNIFORM ILLUMINATION AND ZERO MEAN GAUSSIAN NOISE OF VARIANCE 0.0025

\begin{tabular}{|l|l|l|}
\hline Smoothing filter & PSNR & MSE \\
\hline Global & 48.1788 & 0.9890 \\
\hline Local & 54.9907 & 0.2061 \\
\hline
\end{tabular}

TABLE III: PSNR AND MSE VALUES OF SEGMENTED FREQUENCY-DOMAIN WAVEFORM AFTER SMOOTHED WITH GLOBAL AND LOCAL OPERATORS

\begin{tabular}{|l|l|l|}
\hline $\begin{array}{l}\text { Smoothing } \\
\text { filter }\end{array}$ & PSNR & MSE \\
\hline Global & 48.2857 & 0.96500 \\
\hline Local & 64.0588 & 0.0255 \\
\hline
\end{tabular}

When we compare the level of impact on Time-domain vs Frequency-domain waveform images, it implies that frequency-domain waveform images are much more positively affected by the use of localized smoothing operators. For the waveform images see Fig. 5a and see Fig. $5 \mathrm{~b}$, which are frequency domain and time-domain 
representation of the same signal, the PSNR gap between the results of global smoothing and local smoothing is increased by 8.16 for time domain signal and 15.77 for frequency domain signal. The MSE also shifted by 0.78 for time-domain while it shifted by 0.93 for frequency domain, see Table III, and see Table IV. See Fig. 10 and its corresponding quality metrics tables, also confirm that the use of localized filters on frequency-domain signals have much more enhanced result than applied to time-domain signals.

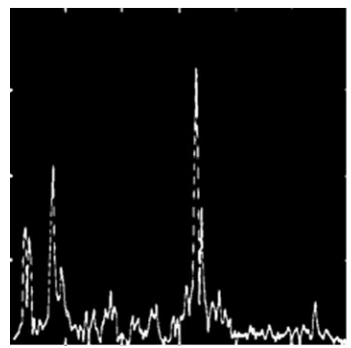

a

Fig. 6. (a) (b) segmented Frequency-domain image in Fig. (5a) after smoothed with global and localized operators, respectively.

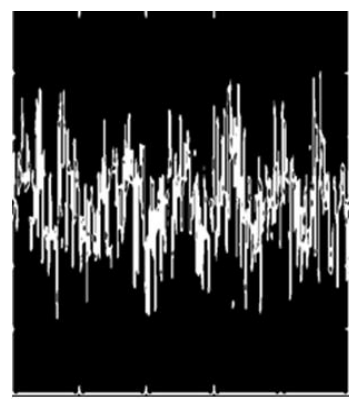

a

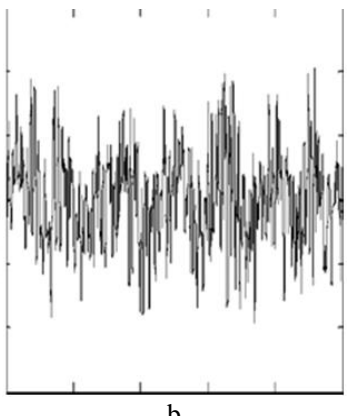

b

Fig. 7. (a) (b) Time-domain waveform image in Fig. (5b) segmented after smoothing using global and local operator, respectively.

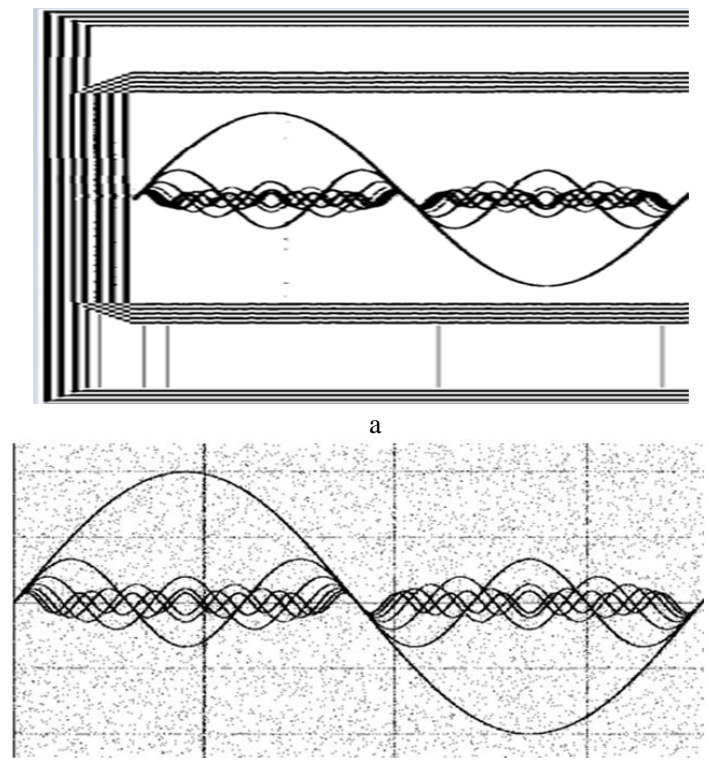

Fig. 8. (a)(b) Segmentation result of Time-domain waveform corrupted with Gaussian noise of zero mean with variance of 0.01 , with global and localized smoothing, respectively.

In general, the experiment results verified that smoothing waveforms with global smoothing filters have a negative impact which cannot be tolerated. The impact is using serious and could lead to misinterpretation of waveforms, in turn, may cause inefficiency of the next stage of image processing system. But it clearly demonstrated the outperforming positive impact of using localized smoothing filters for better result of segmentation of waveform images. It is triggered by the natural behavior of waveform images structure, which is a tiny structure with homogeneous texture. As a result, the segmentation output is supposed to be also a neat, tiny and complete waveform with uniform texture for each input waveform. The use of localized smoothing filters also has shown significant performance increment on the frequency-domain waveform images.

TABLE IV: PSNR AND MSE VALUES OF SEGMENTED TIME-DOMAIN WAVEFORM IMAGE AFTER SMOOTHED WITH GLOBAL AND LOCAL

\begin{tabular}{|l|l|l|}
\multicolumn{3}{c}{ OPERATORS } \\
\hline Smoothing filter & PSNR & MSE \\
\hline Global & 48.9260 & 0.8327 \\
\hline Local & 57.0890 & 0.1271 \\
\hline
\end{tabular}

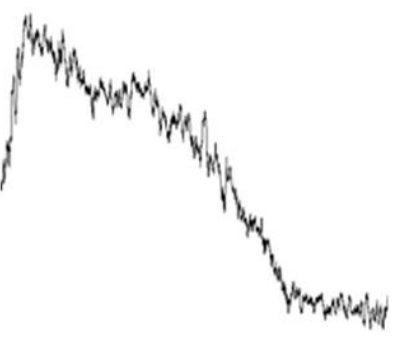

a

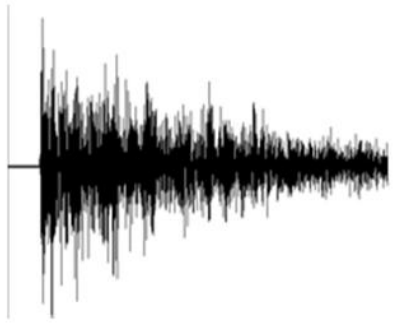

$\mathrm{b}$
Fig. 9. (a) Frequency-domain sound waveform image. (b) Time-domain sound waveform image.

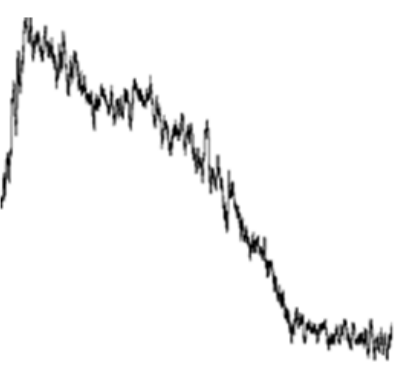

a

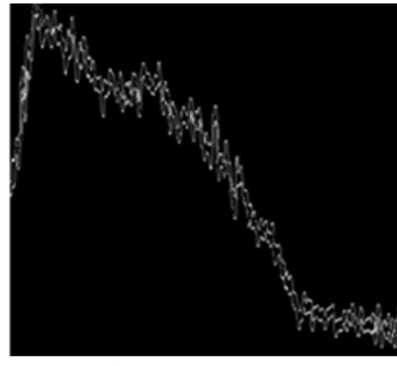

b
Fig. 10. (a)(b) Frequency-domain waveform image in Fig. (9a) segmented after smoothed with localized operator and global operator, respectively.

TABLE V: PSNR AND MSE VALUES OF FREQUENCY-DOMAIN WAVEFORM IMAGE SEGMENTED AFTER SMOOTHED WITH THE LOCALIZED AND GLOBAL

\begin{tabular}{|l|l|l|}
\multicolumn{3}{l}{ OPERATORS } \\
\hline Smoothing type & PSNR & MSE \\
\hline Global & 48.2166 & 0.9804 \\
\hline Local & 61.9699 & 0.0413 \\
\hline
\end{tabular}

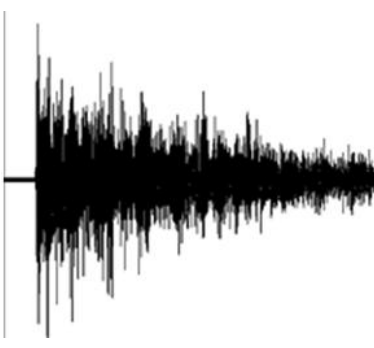

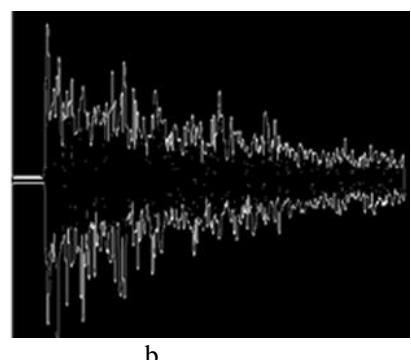

$\mathrm{b}$
Fig. 11. (a)(b) Time-domain waveform image in Fig. (9b) segmented after smoothed with localized operator and global operator, respectively.

TABLE VI: PSNR AND MSE VALUES OF TIME-DOMAIN WAVEFORM IMAGE SEGMENTED AFTER SMOOTHED USING LOCAL AND GLOBAL OPERATORS

\begin{tabular}{|l|l|l|}
\hline Smoothing type & PSNR & MSE \\
\hline Global & 48.3209 & 0.9572 \\
\hline Local & 54.5196 & 0.2297 \\
\hline
\end{tabular}




\section{CONCLUSION}

The contribution of image preprocessing for the success of the next phase of image processing system is significant. Considering the fact we have researched the impact of applying smoothing filters on waveform image segmentation. To identify the impact of smoothing filters on the output of segmentation phase we made experiments on real and synthetic images. We used both ordinary natural images and waveform images. Sample images were smoothed with global smoothing splines and local smoothing splines parameters before segmentation. The smoothed images are segmented using ICM.

During the experiment analysis, results are evaluated using a visual Comparison, PSNR, and MSE. The experimental results confirmed that the general smoothing recommendations made for ordinary images should be filtered out for waveform images because of their unique characteristics. Global smoothing operators applied to ordinary images has shown positive impact and led to perfect segmentation result from image processing point of view. But for waveform images, the global smoothing operators resulted in a negative impact. The structure of the image is affected seriously that led to very poor segmentation result, on the other hand using localized smoothing operators on the waveform has improved the segmentation result sufficiently. The use of localized smoothing filters on frequency-domain waveform images also has shown significant performance improvement than time-domain waveform images.

So we propose to avoid using global smoothing operators for preprocessing of waveform images which have a serious negative impact. We strongly recommend using localized smoothing operators.

\section{REFERENCES}

[1] N. G. Paulterl, D. R. Larson, and J. J. Blair, "The IEEE standard on transitions, pulses, and related waveforms," in Proc. 2003 IEEE Instrument and Measurement Technology Conference(IMTC), USA.

[2] W. C. Cheng and H. H. Tseng, "Removing smoothing effects for color image segmentation," in Proc. 2015 IEEE International Conference on Systems, Man, and Cybernetics, Kowloon, 2015, pp. 2004-2009.

[3] D. S. Prabha and J. S. Kumar, "Performance analysis of image smoothing methods for low level of distortion," in Proc. 2016 IEEE International Conference on Advances in Computer Applications (ICACA), Coimbatore, 2016, pp. 372-376.

[4] C. R. Gonzalez and R. E. Woods, Digital Image Processing, India: Dorling Kindersley (India) Pvt Lt Publications, 2011.

[5] C. R. Gonzalez and R. E. Woods, Digital Image Processing, Pearson Education, Second Edition, 2005.

[6] A. Goshtasby and M. Satter, "An adaptive window mechanism for image smoothing," Computer Vision and Image Understanding, vol. 111, pp. 155-169, 2008.

[7] ISPRS, International Archives of Photogrammetry and Remote Sensing, vol. 33, Part B3, Amsterdam, 2000.

[8] C. H. Reinsch, "Smoothing by spline functions," Numer. Math., vol. 10, pp. 177-183, 1967.
[9] M. Unser, A. Aldroubi, and M. Eden, "B-spline signal processing: Part II - Efficient design and applications," IEEE Trans. Signal Process, vol. 41, no. 2, pp. 834-848, Feb. 1993.

[10] T. Lindblad and J. M. Kinser, Image Processing Using Pulse-Coupled Neural Networks, Springer-Verlag Berlin Heidelberg, Third Edition, 2013.

[11] C. Fowlkes and J. Malik, "Learning to detect natural image boundaries using local brightness, color, and texture cues," IEEE Trans. Pattern Anal. Mach. Intell., vol. 26, no. 5, pp. 530-549, 2004.

[12] W. Zhou, B. C. Alan, S. R. Hamid, and S. P. Eero, "Image quality assessment: From error visibility to structural similarity," IEEE Transactions on Image Processing, vol. 13, Issue 4, pp. 600-612, April 2004.

[13] H.-Y. Yang, X.-Y. Wang, X.-Y. Zhang, and J. Bu, "Color texture segmentation based on image pixel classification," Engineering Applications of Artificial Intelligence, vol. 25, pp. 1656-1669, 2012.

[14] C. Kervrann, "An adaptive window approach for image smoothing and structure preserving," in Proc. the European Conference on Computer Vision (ECCV), Prague, Czech Republic, March 2004.

[15] Y. A. Y. Al-Najjar and D. C Soong, "Comparison of image quality Assessment: PSNR, HVS, SSIM, UIQI," International Journal of Science and Engineering Research, vol. 3, pp. 1-5, 2012.

[16] S. C. W. Cai and D. Zhang, "Fast and robust fuzzy c-means clustering algorithms incorporating local information for image segmentation," Pattern Recognition, vol. 40, no. 3, pp. 825-838, 2007.

[17] I. J. Schoenberg, "Spline functions and the problem of graduation," Proc. Nat. Acad. Sci., vol. 52, pp. 947-950, 1964.

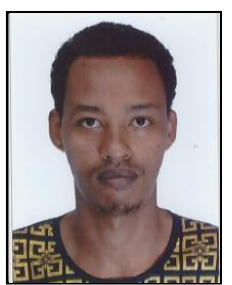

Kedir Kamu Sirur was born in Addis Ababa, Ethiopia in 1985. He received B.Sc. degree in computer science and information technology from Arbaminch University, Ethiopia in 2006, M.Sc. degree in computer science from Addis Ababa University, Ethiopia in 2011. Between 2008 and 2014 he was a lecturer in the Department of Computers Science, in Dilla University, Ethiopia. Now he is a $\mathrm{PhD}$ student in University of Electronic Science and Technology of China, School of Automation Engineering. His research interests include intelligent computing, image processing, and pattern recognition applications.

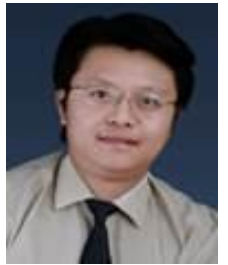

Ye Peng received B.Sc. and Ph.D. degrees in measurement technology and instruments from University of Electronic Science and Technology of China (UESTC) in 1995 and 2010, respectively. He is currently a professor of School of Automation Engineering, in UESTC. His research interests include high-speed data acquisition and signal processing.

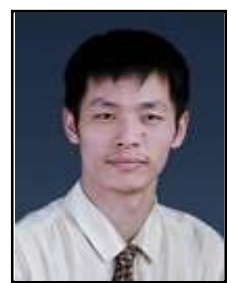

Zhang Qinchuan was born in Chengdu, China, in 1977. He received M.Sc. and Ph.D. degrees from University of Electronic Science and Technology of China (UESTC), in 2004 and 20012, respectively. Now he is an associate researcher in the School of Automation Engineering, in UESTC, China. His research interest includes high-speed data acquisition and signal processing, digital oscilloscope, intelligent computing, etc. 